\title{
Preface to the Special Topic on Modern Acoustics
}

The $9^{\text {th }}$ International Symposium on Modern Acoustics was held in Nanjing, China, on May 19-22, 2012.

In 1985, Prof. WEI RongJue, the pioneer and founder of the Institute of Acoustics of Nanjing University, Member of Chinese Academy of Sciences, organized the First International Workshop on Modern Acoustics. By the end of last century, the Institute of Acoustics had organized eight international workshops with different topics on Modern Acoustics. The workshops provided open forums for worldwide participants to exchange the achievements and experiences in the research of acoustics, which promoted the acoustic research work of China.

The $9^{\text {th }}$ International Symposium was a continuation and development of the workshop series, organized by the Key Laboratory of Modern Acoustics and Institute of Acoustics of Nanjing University, the Acoustical Society of China, The Acoustical Society of Jiangsu Province, and also the Key Laboratory of Underwater Acoustic Signal Processing of Southeast University of China. The Organizing Committee is strongly supported by the Ministry of Education and the National Natural Science Foundation of China and is very grateful to the support and assistance from colleagues and friends, especially from the invited speakers, and also to the key sponsorship from AAC Technologies Holdings Inc. and other companies of related science and technology.

Compared with previous workshop series, the $9^{\text {th }}$ Symposium expanded its scope: the Organizing Committee received about 140 abstracts and 70 full manuscripts, and about 240 participants came from foreign countries and domestic institutions. The topics of the abstracts include Physical Acoustics, Photo-acoustics, Ultrasonics, Biomedical Ultrasonics, Audio Acoustics, Acoustic Signal Processing and Underwater Acoustics.

To extend the influence of the symposium, the Organizing Committee intended to have 50 full reports, peer reviewed, to be published in the special issues of Science China-Physics, Mechanics \& Astronomy and Journal of Nanjing University (Natural Sciences) (Vol 48(5), 2012 and Vol. 49(1), 2013) separately. In the special topic of Science China-Physics, Mechanics \& Astronomy, seventeen papers on six aspects including physical acoustics, ultrasonics, photoacoustics, biomedical ultrasonics, underwater acoustics and audio acoustics were selected and introduced below.

(1) Physical acoustics: 1) Theoretical study of the ultrasonic radiation force acting on particles caused by focusing ultrasound is presented based on the ray acoustic approach and the ultrasound attenuation [1];2) The distributions of velocity fields caused by nonlinear vibrations of a couple of bubbles in ultrasonic field is studied theoretically, and the symmetry of the tangential velocity distribution implies a possibility of attraction or repulsion of the bubble pairs [2] ; 3) A multi-objective optimization approach is presented to design a one-dimensional phonon-crystal structure based on the band gaps mechanism and the intelligent algorithm [3].

(2) Ultrasonics: 1) A method to evaluate frequency-dependent coupling-of-modes parameters is proposed to optimize the design of SAW devices, which utilizes the SAW field distributions in the finite periodic metal gratings calculated by FEM/BEM [4]; 2) Air-coupled ultrasonic transducers are used for delamination detection in laminated composite beams, and the mechanisms of interaction between $A_{0}$ mode of the Lamb wave and delamination are studied [5]; 3) The preparation of lanthanide (III) doped zinc oxide nanoparticles in room-temperature ionic liquid via an ultrasonic irradiation has been studied, and a possible mechanism is proposed to explain the results [6].

(3) Photoacoustics: 1) Photoacoustic investigation of different $\mathrm{TiO}_{2}$ thin films on $\mathrm{Si}$ substrates is presented experimentally and theoretically, and the attention is paid to finding the best experimental conditions of elastic bending detection method [7]; 2) The ultra-shallow junctions of As+ ion implantated and spike annealed Si wafers are characterized non-destructively and effectively by spectroscopic ellipsometry, photocarrier radiometry and photoluminescence techniques [8]; 3) Photolyses of carboxy-hemoglobin of several mammals are investigated by photoacoustic calorimetry, and the dynamic parameters of enthalpy and conformational volume changes in the photolyses are obtained [9].

(4) Biomedical ultrasonics: 1) An explicit standard for the selection of the signal-of-interest in ultrasonic backscattering 
techniques is proposed for studying cancellous bones [10]; 2) A joint spectrogram segmentation and ridge-extraction method is proposed to separate multiple modes of ultrasonic guided waves in long bones, by which the reconstructed signals are highly related to the original signals [11]; 3) The extended two-mass model is adopted to analyze the nonlinear oscillation of pathological vocal folds, which indicates that the pathological vocal-fold decreases the fundamental frequency and eliminates the high-order harmonics [12].

(5) Underwater acoustics: 1) A fast algorithm based on clustering search to reduce the computation of steered response power-phase transform is proposed for sound source localization, by which the computational load is reduced greatly [13]; 2) An intrinsic mode characteristic extraction method is presented to analyze underwater cylindrical shell acoustic radiations based on the mechanism of shell vibration, and then to identify effectively the shell scale parameters [14]; 3) An underwater 3-D acoustic imaging approach based on a narrowband multiple-input and multiple-output array is proposed, which not only saves a great number of sensors, but also halves the array size [15].

(6) Audio acoustics: 1) A model to analyze nonlinear parameters of large-amplitude vibrations of loudspeakers is introduced, which can predict the performance and identify the defects of the loudspeakers [17]; 2) The nonlinear properties of suspensions of electrodynamic loudspeakers are studied theoretically and experimentally, so that the performance of loudspeakers, such as harmonic distortion, can be predicted and reduced [18].

Generally, the papers collected in this topic carry new ideas in the related aspects of modern acoustics, which will turn to breed new findings and promote further exchange in acoustic research. Finally, on behalf of the Organizing Committee, we would like to express our gratitude to all authors, reviewers and editors for their contributions to the special topic.

\section{Professors ZHANG ShuYi, QIU XiaoJun \& LIU XiaoZhou Guest Editors Key Laboratory of Modern Acoustics, Institute of Acoustics, Nanjing University}

1 Wu R R, Liu X Z, Gong X F. A study of the acoustical radiation force considering attenuation. Sci China-Phys Mech Astron, 56(7): 1237-1245

2 Wang C H, Cheng J C. The velocity field around two interacting cavitation bubbles in an ultrasound field. Sci China-Phys Mech Astron, 56(7): 1246-1252

3 Zhang P, Wang Z Y, Zhang Y Q, et al. Multi-band design for one-dimensional phononic crystals. Sci China-Phys Mech Astron, 56(7): 1253-1262

4 Wang H, Wang W B, Wu H D, et al. Precise simulation of surface acoustic wave devices using frequency-dependent coupling-of-modes parameters. Sci China-Phys Mech Astron, 56(7): 1263-1268

5 Liu Z H, Yu H T, He C F, et al. Delamination damage detection of laminated composite beams using air-coupled ultrasonic transducers. Sci China-Phys Mech Astron, 56(7): 1269-1279

6 Gao B, Yang Y T, Yang H, et al. Study of lanthanide doped zinc oxide nanoparticles synthesized via a sonochemical method. Sci China-Phys Mech Astron, 56(7): 1280-1284

7 Todorovi D, Rabasovi M D, Markushev D, et al. Study of $\mathrm{TiO}_{2}$ thin films on Si substrate by the photoacoustic elastic bending method. Sci China-Phys Mech Astron, 56(7): 1285-1293

8 Huang Q P, Li B C, Ren S D. Optical and photo-carrier characterization of ultra-shallow junctions in silicon. Sci China-Phys Mech Astron, 56(7): 1294-1300

9 Zhao J Y, Li J H, Zhang Z, et al. Photolyses of mammalian carboxy-hemoglobin studied by photoacoustic calorimetry. Sci China-Phys Mech Astron, 56(7): 1301-1309

10 Liu C C, Han H J, Ta D A, et al. Effect of selected Signals of Interest on ultrasonic backscattering measurement in cancellous bones. Sci China-Phys Mech Astron, 56(7): 1310-1316

11 Zhang Z G, Xu K L, Ta D A, et al. Joint spectrogram segmentation and ridge-extraction method for separating multimodal guided waves in long bones. Sci China-Phys Mech Astron, 56(7): 1317-1323

12 Wan N, Peng D D, Sun M, et al. Nonlinear oscillation of pathological vocal folds during vocalization. Sci China-Phys Mech Astron, 56(7): 1324-1328

13 Zhao X Y, Tang J, Zhou L, et al. Accelerated steered response power method for sound source localization via clustering search. Sci China-Phys Mech Astron, 56(7): 1329-1338

14 Liu Q Y, Fang S L, Cheng Q, et al. Intrinsic mode characteristic analysis and extraction in underwater cylindrical shell acoustic radiation. Sci ChinaPhys Mech Astron, 56(7): 1339-1345

15 Liu X H, Sun C, Yi F, et al. Underwater three-dimensional imaging using narrowband MIMO array. Sci China-Phys Mech Astron, 56(7): 1346-1354

16 Heng W, Shen Y, Xia J, et al. Analysis and prediction of loudspeaker large-signal symptoms. Sci China-Phys Mech Astron, 56(7): 1355-1360

17 Feng Z X, Shen Y, Heng W, et al. Nonlinear behavior of electrodynamic loudspeaker suspension at low frequencies. Sci China-Phys Mech Astron, 56(7): 1361-1365 